\title{
A microRNA component of the hypoxic response
}

\author{
R Kulshreshtha ${ }^{1}$, RV Davuluri ${ }^{2}$, GA Calin ${ }^{3}$ and M Ivan ${ }^{\star, 1}$
}

microRNAs participate in a wide variety of physiological and pathological cellular processes. Recent studies have established a link between a specific group of microRNAs and hypoxia, a key feature of the neoplastic microenvironment. A significant proportion of the hypoxia-regulated microRNAs (HRMs) are also overexpressed in human cancers, suggesting a role in tumorigenesis. Preliminary evidence suggests that they could affect important processes such as apoptosis, proliferation and angiogenesis. Several HRMs exhibit induction in response to HIF activation, thus extending its repertoire of targets beyond translated genes. In the present review, we discuss the emerging roles of HRMs in oxygen deprivation in cancer context.

Cell Death and Differentiation (2008) 15, 667-671; doi:10.1038/sj.cdd.4402310; published online 25 January 2008

\section{Small Size Regulators with Far-Reaching Impact}

MicroRNAs are currently thought to regulate the expression of most genes and consequently play critical roles in the coordination of a wide variety of processes, including differentiation, proliferation, death and metabolism. ${ }^{1-3}$ They exert their actions at post-transcriptional level, either via translational repression and/or mRNA degradation. ${ }^{4,5}$

As the 'noncoding RNA revolution' continues to unfold, a large number of microRNAs have been associated with tumorigenesis and suspected to mechanistically participate in this complex process. Thus, specific microRNA patterns have been described in tumors, and in some cases shown to correlate with their clinico-pathological features. Additionally, a significant number of microRNAs are encoded within fragile sites, regions of amplification, or common breakpoint regions associated with human cancers. ${ }^{6-12}$

\section{Regulatory Mechanisms of microRNA Expression: the Role of Hypoxia}

The information about specific regulation of microRNAs has comparatively lagged behind, in contrast to the wealth of publications about their biological effects. Recent studies showed that select microRNAs can be controlled by transcription factors involved in the regulation of 'classic genes' in response to various endogenous and exogenous stimuli. For example, the oncogene product and transcription factor $c-M Y C$ activates the miR-17-92 cluster, and this mechanism plays an important role in tumor formation. ${ }^{13}$ Similarly, E2F transcription factor family was also found to regulate this oncogenic cluster. ${ }^{14}$ Thus, it is conceivable that engaging a microRNA component is a more general feature of transcription factors action, and recent work addressed such a mechanism in the context of the hypoxia response.

Hypoxia is an essential feature of the neoplastic microenvironment. Tumors with extensive low oxygen tension tend to exhibit poor prognosis and resistance to conventional therapy. The molecular mechanisms of response to hypoxia are extremely complex, a key role being played by a transcriptional regulator, hypoxia-inducible factor (HIF), which orchestrates the expression of a wide variety of genes thought to be critical for adaptation to low oxygen. ${ }^{15-20}$

While specific gene induction by low oxygen has arguably dominated hypoxia research, more recently the study of gene repression by hypoxia has received increasing attention. One of the interesting features of the latter process is its relative selectivity. Thus, a large percentage of genes continue to be expressed at quasi-normoxic levels, while the translation/ transcription of others is significantly suppressed. Our working hypothesis has been that microRNAs could be a part of this process (Figure 1).

Studies from our group identified a set of hypoxia-regulated microRNAs (HRMs), providing an additional link between a tumor-specific stress factor and gene expression control. The HRM group includes: miR-21, 23a, 23b, 24, 26a, 26b, 27a, 30b, 93, 103, 103, 106a, 107, 125b, 181a, 181b, 181c, 192, 195, 210 and 213, which were consistently induced in response to hypoxia in the breast and colon cancer cells tested. ${ }^{21}$ Our study selected only microRNAs exhibiting consistent upregulation in at least two cell lines and at several time points in hypoxia, potentially increasing the stringency of the screen. Three additional articles reported microRNAs that respond to low oxygen with some notable similarities, including miR-210, miR-30b, 93 and 181b. ${ }^{22-24}$

It is also true, however, that a significant number of microRNAs differed between the studies, which is not

\footnotetext{
${ }^{1}$ Molecular Oncology Research Institute, Tufts-New England Medical Center, Boston, MA 02111, USA; ${ }^{2}$ Comprehensive Cancer Center, Ohio State University, Columbus, OH 43210, USA and ${ }^{3}$ Experimental Therapeutics Department, MD Anderson Cancer Center, Houston 77030, TX, USA

${ }^{*}$ Corresponding author: M Ivan, Molecular Oncology Research Institute, Tufts-New England Medical Center, 750 Washington Street, Box 5609, Boston, MA 2111, USA. Tel: + 617636 7514; Fax: +617 636 6127; E-mail: mivan@tufts-nemc.org

Keywords: microRNA; hypoxia; cancer; regulation; expression profiles; target genes

Abbreviations: AMO, antimicroRNA oligonucleotides; HIF, hypoxia-inducible factor; HRM, hypoxia-regulated microRNAs; LNA, locked nucleic acids

Received 17.10.07; revised 11.12.07; accepted 12.12.07; Edited by N Chandel; published online 25.1.08
} 


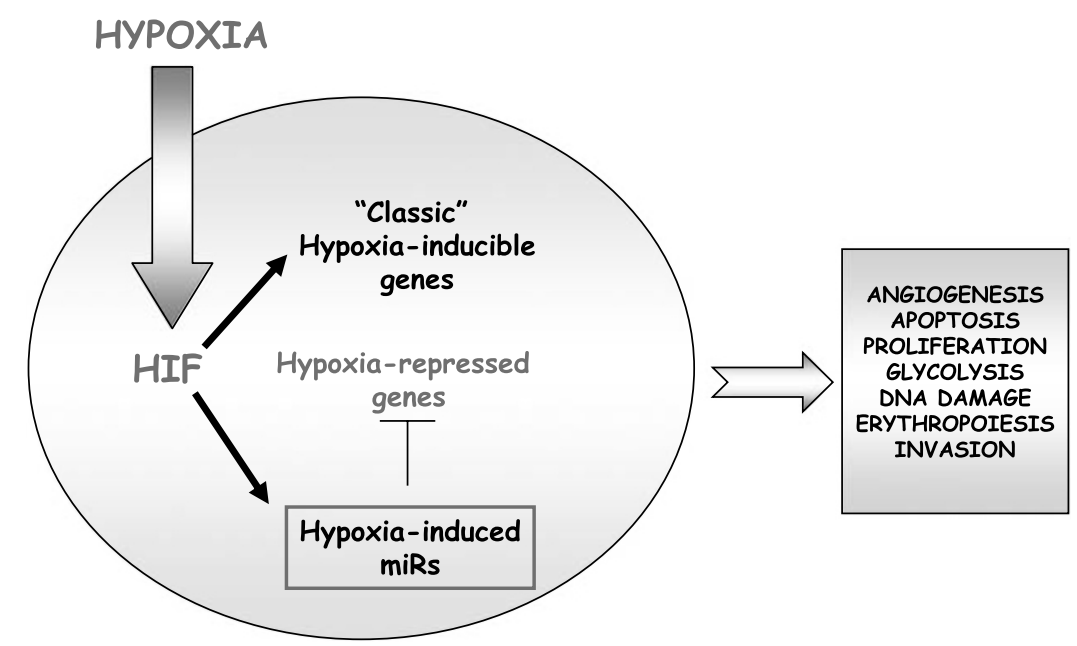

Figure 1 Proposed model implicating select microRNAs in the hypoxia response

necessarily surprising, given the differences in the cellular backgrounds and technology employed, both being a recognized source of variability. An additional difference was in the experimental conditions employed by the different groups: hypoxia mimetics, ${ }^{22}$ versus $1 \%$ oxygen for $1 \mathrm{~h},{ }^{23}$ versus $5 \%$ oxygen for $8 \mathrm{~h},{ }^{23}$ versus $0.2 \%$ for various periods of time from $8-48$ h. $^{21}$

In addition to the microRNAs that respond to hypoxia by upregulation, a set of microRNAs were identified as downregulated in hypoxic cells, including miR-15b, 16, 19a, 20a, 20b, 29b, 30b, 30e-5p, 101, 141, 122a, 186, 320 and $197 .{ }^{22-24}$ In our study, we have also detected microRNAs that exhibited downregulation at the level of microarrays (miR-126, 128, $138,323,326)$; however, the changes seemed restricted to one cell line and were not pursued at this stage (R Kulshreshtha et al., unpublished).

An interesting case is represented by the members of let- 7 family, which seem to exhibit contrasting patterns of response during hypoxia, with the caveat that the findings were reported in different cell types and by different groups. Thus, let-7g, let-7e and let-7i were identified as hypoxia-inducible whereas let-7a, $c, d, e, f$ and $g$ levels decreased during hypoxia exposure. $^{22,23}$ In our hands, several let-7 forms (f, $\left.g, i\right)$ exhibited contrasting changes in different colon and breast cancer cell lines ( $R$ Kulshreshtha et al., unpublished), suggesting that the let-7 family could contain microRNAs that respond to hypoxia in a more cell-specific manner. A summary of all the microRNAs reported as hypoxia-responsive (either by induction or repression) is provided in Table 1 (for more detailed information, see Supplementary Tables 1 and 2).

Our study experimentally confirmed an important regulatory role for HIF, at least for some hypoxia-induced microRNAs, such as miR-210, 26 and 181. The strategy employed a combination of HIF transduction, chromatin immunoprecipitation and luciferase-based reporters driven by fragments of select HRM promoters. ${ }^{21}$

Delineating the promoter regions of microRNAs, where relevant transcription factors such as HIF bind, is a necessary step for an expanded understanding of microRNA expression control. The main challenge comes from the fact that only few microRNA promoters have been identified experimentally. ${ }^{25-27}$
In a preliminary analysis of the promoters of all known and predicted microRNAs, we predicted HIF-binding sites by position weight matrix approach. ${ }^{21}$ While our methodology analyzed the $5 \mathrm{~kb}$ promoter region of all the microRNAs, the promoter regions can span much longer regions. The analysis, performed separately for individual types of HIFbinding consensuses (V\$HF_Q3 and V\$HIF_Q5) revealed that the HRMs (as a group) contains significantly more microRNAs with at least one HIF site than the average random 23 microRNAs.

Additionally, approximately $6 \%$ of the human microRNAs exhibit HIF sites significantly conserved across 17 species, which could reflect functional importance. ${ }^{28}$ Such searches could help us identify additional HRMs, which were likely missed by the original array-based screen.

Recently, additional transcription factors that under certain conditions respond to hypoxia/anoxia, such as p53 and $\mathrm{NF}-\kappa \mathrm{B}$, have been shown to affect the expression of select microRNAs. ${ }^{29,30}$ It is however premature to state whether or not such pathways could play significant role in the hypoxic response.

We also identified additional candidate transcription factor sites in the proximity of select HRM, some of them exhibiting a high degree of evolutionary conservation, suggesting a biologically important role. An example for miR-210 is given in Figure 2, showing candidate sites for several transcription sites (Oct-C, AP2, PPAR $\gamma$ and E2F) which could potentially regulate its expression as part of the hypoxia response or in response to unrelated stimuli.

\section{Towards Identification of Cellular HRM Targets}

Identification of targets with biological impact remains without doubt a highly complex endeavor in the study of microRNAs. Several programs for target gene prediction are currently available, such as PicTar (pictar.bio.nyu.edu), TargetScan (www.targetscan.com) and miRBase (http://microrna.sanger. ac.uk/cgi-bin/targets/v1/search.pl). ${ }^{31-33}$ They employ different algorithms and ranking criteria and are known to produce only a partially overlapping set of candidates. 
Table 1 Compilation of microRNAs associated with the hypoxia response by recent publications

\begin{tabular}{|c|c|c|c|}
\hline \multicolumn{2}{|c|}{$\begin{array}{l}\text { MicroRNAs upregulated } \\
\text { by hypoxia }\end{array}$} & \multicolumn{2}{|c|}{$\begin{array}{l}\text { MicroRNAs downregulated } \\
\text { by hypoxia }\end{array}$} \\
\hline Mir-7 & 3 & Mir-15b & 2 \\
\hline Mir-15a & 3 & Mir-16 & 2 \\
\hline mir-21 & 1 & Mir-19a & 3 \\
\hline mir-23a & 1 & Mir-20a & 2 \\
\hline Mir-23b & 1 & Mir-20b & 2 \\
\hline Mir-24 & 1 & Mir-26b & 2 \\
\hline Mir-26a & 1 & Mir-29b & 3 \\
\hline Mir-26b & 1 & Mir-30b & 2 \\
\hline Mir-27a & 1 & Mir-30e-5p & 3 \\
\hline Mir-30b & 1,3 & Mir-101 & 3 \\
\hline Mir-30d & 2 & Mir-122a & 3 \\
\hline Mir-93 & 1,4 & Mir-141 & 3 \\
\hline Mir-98 & 3 & Mir-186 & 3 \\
\hline Mir-103 & 1 & Mir-195 & 3 \\
\hline Mir-106a & 1 & Mir-197 & 3 \\
\hline Mir-107 & 1 & Mir-224 & 2 \\
\hline Mir-125b & 1 & Mir-320 & 3 \\
\hline Mir-148a & 3 & Mir-374 & 3 \\
\hline Mir-148b & 3 & Mir-422b & 3 \\
\hline Mir-151 & 2 & Mir-424 & 3,4 \\
\hline Mir-155 & 2 & Mir-565 & 3 \\
\hline Mir-181a & 1 & Let-7-a & 2 \\
\hline Mir-181b & 1,2 & Let-7-c & 2 \\
\hline Mir-181c & 1 & Let-7-d & 2 \\
\hline Mir-188 & 2 & Let-7-e & 2 \\
\hline Mir-191 & 3 & Let-7-f & 2 \\
\hline Mir-192 & 1 & Let-7-g & 2 \\
\hline Mir-195 & 1 & & \\
\hline Mir-200a & 3 & & \\
\hline Mir-210 & $1,2,3$ & & \\
\hline Mir-213 & 1 & & \\
\hline Mir-214 & 3 & & \\
\hline Mir-373 & 3 & & \\
\hline Mir-429 & 3 & & \\
\hline Mir-498 & 3 & & \\
\hline Mir-563 & 3 & & \\
\hline Mir-572 & 3 & & \\
\hline Mir-628 & 3 & & \\
\hline Mir-637 & 3 & & \\
\hline Let-7-e & 3 & & \\
\hline Let-7-g & 3 & & \\
\hline Let-7-i & 3 & & \\
\hline
\end{tabular}

Study cited, and corresponding cell types and conditions: (1) Kulshreshtha et al., 2007 - colon and breast cancer cells, $0.2 \%$ oxygen for $8-48 \mathrm{~h}$. (2) Hua et al., 2006 - Nasopharyngeal carcinoma cell line, DFOM treatment for $20 \mathrm{~h}$. (3) Hebert et al., 2007 - Head and neck Squamous cell carcinoma, 1 or $5 \%$ oxygen for 1 and $8 \mathrm{~h}$, respectively. (4) Donker et al., 2007 - Primary human cytotrophoblasts, $<1 \%$ oxygen for $48 \mathrm{~h}$

In the case of hypoxia-regulated microRNAs, in silico searches reveal a highly complex spectrum of candidate targets, including genes involved in proliferation, apoptosis, DNA repair, chromatin remodeling, metabolism and migration. Each HRM is predicted to downregulate in excess of 10 genes, sometimes as many as 200 , which could confound the effort to identify biologically relevant targets.

One set of targets worth pursuing is cell death regulators, given the importance of this process in a stressful environment, such as hypoxia. Using the available prediction programs, several key genes of the apoptotic response were found to be potentially targeted by HRMs: PAR-4 (miR-26, 30, 181), PCDC10 (miR-103/107, miR-181), BID (miR-23), BIM (miR-24); CASP3 (miR-30), CASP 7 (miR-23), APAF1 (miR-27), BAK1 (miR-26), Bnip3L (miR-23) (Figure 3). Conversely, one of the best-documented antiapoptotic genes (Bcl2) is an experimentally-confirmed target of miR15 and 16. These microRNAs were found to respond to hypoxia by downregulation, at least in one cell type. ${ }^{22,34}$ One could therefore predict a mechanism whereby an increase in $\mathrm{Bcl} 2$ in hypoxia occurs, in part, by microRNA downregulation.

We do not imply, however, that HRMs exhibit a general antiapoptotic effect in hypoxia. Indeed, it is entirely conceivable that any given HRM could feed into downstream pathways containing both pro- and antiapoptotic genes. Which side the balance will shift could depend on a variety of factors, including cellular context and additional stimuli.

Another process known to be affected by hypoxia is proliferation, since many cell types undergo cell cycle slowdown or arrest during oxygen deprivation. A multitude of cell cycle genes are in silico HRMs targets, a few examples being: cdc25A (miR-21, miR-103/107), cyclin D2 (miR-26, miR-103/ 107), cyclin E1 (miR-26), cyclin H (miR-23), cdk6 (miR-26, $\mathrm{miR}-103 / 107)$. One possibility that remains to be tested is that coordinated induction of HRMs in hypoxia exhibits an independent regulatory impact on cell cycle.

A potential microRNA target of particular importance is VEGF, arguably the most studied angiogenic factor and wellestablished therapy target. For this gene, a group of candidate regulatory microRNAs have been identified recently: miR-16, miR-20, let-7b, miR-17-5p, miR-27, miR-106, miR-107, miR-193, miR-210, miR-320 and miR-361. ${ }^{22}$ Interestingly, most of these microRNAs were found to respond to hypoxia, which could lead to an extra layer of complexity in the angiogenic response.

The number and variety of targets for each miR raises an experimental challenge, one prediction being that manipulation of any individual target will fail to fully capture the phenotypic impact of the corresponding miR in low oxygen. Moreover, it is entirely logical that experimental manipulation of any given microRNA in hypoxia will fail to reproduce the effect of coordinated changes of all the HRMs.

\section{Towards Future Applications in Clinical Oncology}

Recent investigations have dissected a large number of cancers (breast, lung, colon, stomach, prostate carcinomas and pancreatic endocrine tumors) for microRNA expression and identified specific alterations compared to normal cells. ${ }^{35}$ Interestingly, the majority of HRMs are also overexpressed in at least some types of tumor types, suggesting that hypoxia represents a contributing element for microRNA alterations in cancer.

The patterns of microRNA alterations reported in cancer versus normal tissues is very likely the sum of a large variety of highly complex molecular signals, including activation of oncogenic pathways and microenvironmental factors (hypoxia, pH alterations). Given the relatively low number of microRNAs compared to the 'conventional genes', one could anticipate that microRNA profiles could help provide a readout of the activated signaling pathways in individual tumors. However, for such an effort to become feasible, a detailed understanding of the tumor-relevant regulators is of utmost importance. 


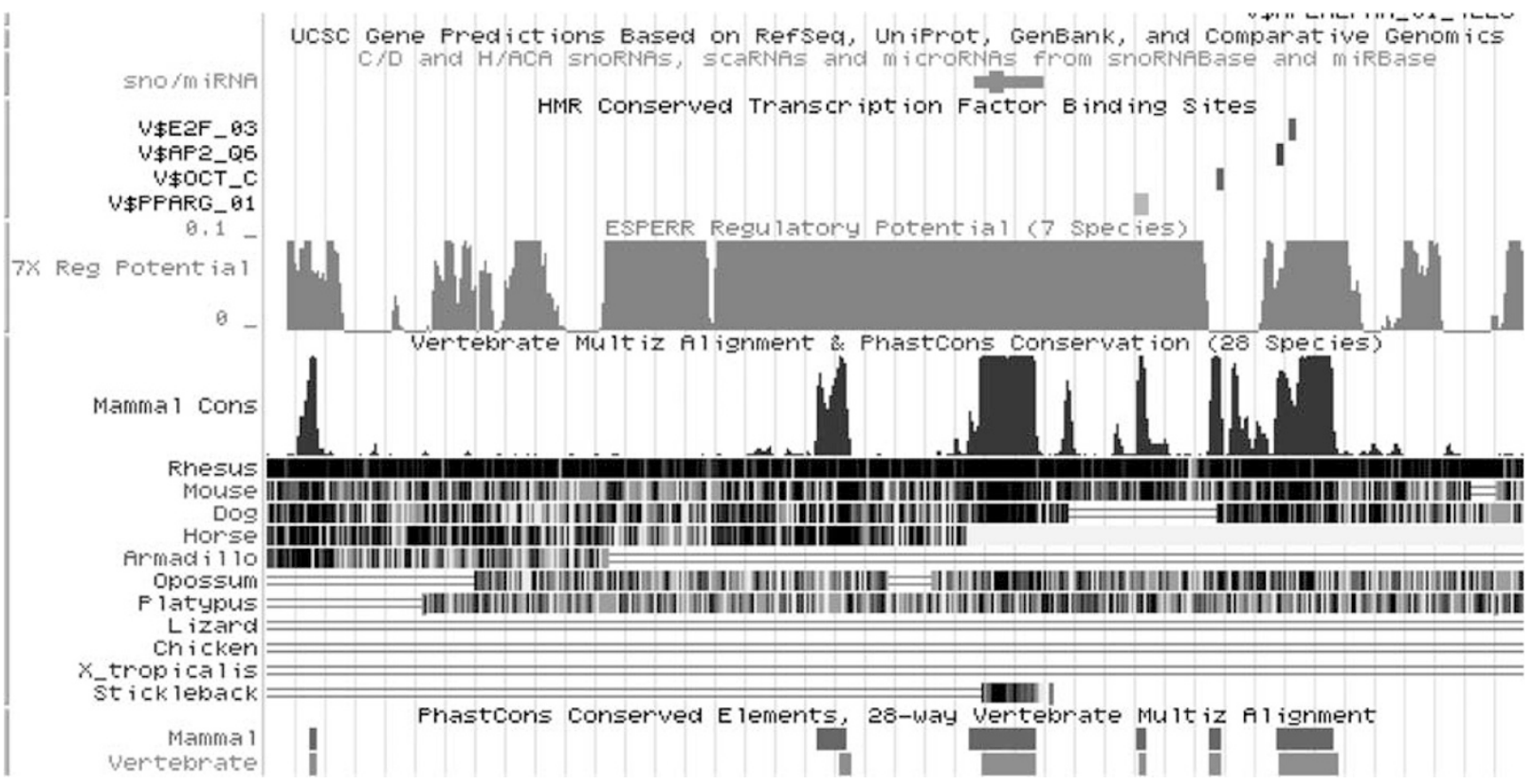

Figure 2 Candidate miR-210 transcriptional regulators, rendered using the UCSD Genome Browser and the TransFac 7.0 (www.gene-regulation.com/pub/ databases.html). Shown are only the transcription factor sites that exhibit significant conservation across species (E2F, AP2, Oct and PPAR $\gamma$ ), suggesting a biological role

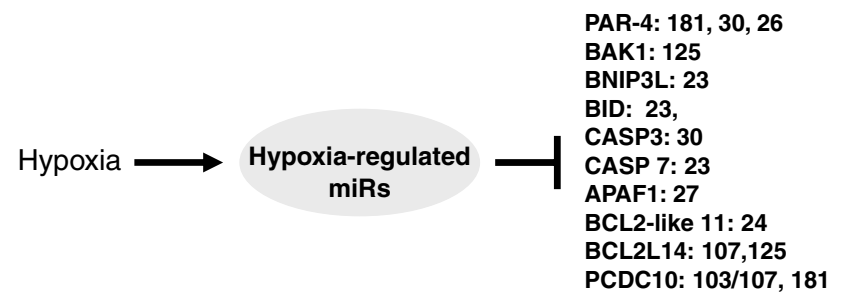

Figure 3 Selection of in silico HRM targets associated with apoptosis (the official gene symbols were used)

Another direction of microRNA research in a tumor context is towards novel pharmacologic approaches. Although the traditional drug targets have been protein products, the recent development of microRNA derivatives with increased stability and binding efficiency, such as AMOs (antimicroRNA oligonucleotides) and LNAs (locked nucleic acids) represent potentially important developments for such purpose. ${ }^{36-38}$ For example, targeting an HRM that plays a survival role in hypoxia could provide a new angle in targeting a notoriously refractory fraction of tumor cells. Moreover, manipulation of select microRNAs could synergize with conventional therapies. For example, overexpression of miR-21 (identified as HRM), enhances the effect of gemcitabine on cholangiocarcinoma cells. ${ }^{39}$

Acknowledgements. This work was supported by the NIH Grant P30 DK34928 and AACR/PanCan career development award to Ml; Kimmel Scholar award to GAC; RD is supported by grants from National Cancer Institute (Project 3 of U54CA113001), National Human Genome Research Institute (R01HG003362) and American Cancer Society (RSG-06-268-01).

1. Karp X, Ambros V. Developmental biology. Encountering microRNAs in cell fate signaling Science 2005; 310: 1288-1289.
2. Miska EA. How microRNAs control cell division, differentiation and death? Curr Opin Genet Dev 2005; 15: 563-568.

3. Kloosterman WP, Plasterk RH. The diverse functions of microRNAs in animal development and disease. Dev Cell 2006; 11: 441-450.

4. Bartel DP. MicroRNAs: genomics, biogenesis, mechanism, and function. Cell 2004; 116: 281-297.

5. Rana TM. Illuminating the silence: understanding the structure and function of small RNAs. Nat Rev Mol Cell Biol 2007; 8: 23-36.

6. Calin GA, Ferracin M, Cimmino A, Di Leva G, Shimizu M, Wojcik SE et al. A microRNA signature associated with prognosis and progression in chronic lymphocytic leukemia. N Engl J Med 2005; 353: 1793-1801.

7. Croce CM, Calin GA. miRNAs, cancer, and stem cell division. Cell 2005; 122: 6-7.

8. He L, Thomson JM, Hemann MT, Hernando-Monge E, Mu D, Goodson S et al. A microRNA polycistron as a potential human oncogene. Nature 2005; 435: 828-833.

9. Iorio MV, Ferracin M, Liu CG, Veronese A, Spizzo R, Sabbioni $S$ et al. MicroRNA gene expression deregulation in human breast cancer. Cancer Res 2005; 65: 7065-7070.

10. Liu CG, Calin GA, Meloon B, Gamliel N, Sevignani C, Ferracin M et al. An oligonucleotide microchip for genome-wide microRNA profiling in human and mouse tissues. Proc Natl Acad Sci USA 2004; 101: 9740-9744.

11. Lu J, Getz G, Miska EA, Alvarez-Saavedra E, Lamb J, Peck D et al. MicroRNA expression profiles classify human cancers. Nature 2005; 435: 834-838.

12. Yanaihara N, Caplen N, Bowman E, Seike M, Kumamoto K, Yi M et al. Unique microRNA molecular profiles in lung cancer diagnosis and prognosis. Cancer Cell 2006; 9: 189-198.

13. O'Donnell KA, Wentzel EA, Zeller KI, Dang CV, Mendell JT. c-Myc-regulated microRNAs modulate E2F1 expression. Nature 2005; 435: 839-843.

14. Woods K, Thomson JM, Hammond SM. Direct regulation of an oncogenic micro-RNA cluster by E2F transcription factors. J Biol Chem 2007; 282: 2130-2134.

15. Harris AL. Hypoxia - a key regulatory factor in tumour growth. Nat Rev Cancer 2002; 2 38-47.

16. Bacon AL, Harris AL. Hypoxia-inducible factors and hypoxic cell death in tumour physiology. Ann Med 2004; 36: 530-539.

17. Gruber M, Simon MC. Hypoxia-inducible factors, hypoxia, and tumor angiogenesis. Curr Opin Hematol 2006; 13: 169-174.

18. Kim JW, Tchernyshyov I, Semenza GL, Dang CV. HIF-1-mediated expression of pyruvate dehydrogenase kinase: a metabolic switch required for cellular adaptation to hypoxia. Cell Metab 2006; 3: 177-185.

19. Koumenis C. ER stress, hypoxia tolerance and tumor progression. Curr Mol Med 2006; 6 : $55-69$

20. Gordan JD, Simon MC. Hypoxia-inducible factors: central regulators of the tumor phenotype. Curr Opin Genet Dev 2007; 17: 71-77.

21. Kulshreshtha R, Ferracin M, Wojcik SE, Garzon R, Alder H, Agosto-Perez FJ et al. A microRNA signature of hypoxia. Mol Cell Biol 2007; 27: 1859-1867.

22. Hua Z, Lv Q, Ye W, Wong CK, Cai G, Gu D et al. MiRNA-directed regulation of VEGF and other angiogenic factors under hypoxia. PLOS ONE 2006; 1: e116. 
23. Hebert C, Norris K, Scheper MA, Nikitakis N, Sauk JJ. High mobility group A2 is a target for miRNA-98 in head and neck squamous cell carcinoma. Mol Cancer 2007; 6: 5.

24. Donker RB, Mouillet JF, Nelson DM, Sadovsky Y. The expression of Argonaute2 and related microRNA biogenesis proteins in normal and hypoxic trophoblasts. Mol Hum Reprod 2007; 13: 273-279.

25. Cai $\mathrm{X}$, Hagedorn $\mathrm{CH}$, Cullen BR. Human microRNAs are processed from capped, polyadenylated transcripts that can also function as mRNAs. RNA 2004; 10: 1957-1966.

26. Zhou X, Ruan J, Wang G, Zhang W. Characterization and identification of microRNA core promoters in four model species. PLoS Comput Biol 2007; 3: e37.

27. Davuluri RV, Grosse I, Zhang MQ. Computational identification of promoters and first exons in the human genome. Nat Genet 2001; 29: 412-417.

28. Kulshreshtha R, Ferracin M, Negrini M, Calin GA, Davaluri RV, Ivan M. Regulation of microRNA expression: The hypoxic component. Cell Cycle 2007; 6: 1426-1431.

29. He L, He X, Lim LP, de Stanchina E, Xuan Z, Liang Y et al. A microRNA component of the p53 tumour suppressor network. Nature 2007; 447: 1130-1134.

30. Kluiver J, van den Berg A, de Jong D, Blokzijl T, Harms G, Bouwman E et al. Regulation of pri-microRNA BIC transcription and processing in Burkitt lymphoma. Oncogene 2007; 26: 3769-3776.

31. Krek A, Grun D, Poy MN, Wolf R, Rosenberg L, Epstein EJ et al. Combinatorial microRNA target predictions. Nat Genet 2005; 37: 495-500.
32. Lewis BP, Shih $\mathrm{IH}$, Jones-Rhoades MW, Bartel DP, Burge CB. Prediction of mammalian microRNA targets. Cell 2003; 115: 787-798.

33. Griffiths-Jones S, Grocock RJ, van Dongen S, Bateman A, Enright AJ. miRBase: microRNA sequences, targets and gene nomenclature. NAR 2006; 34 (Database issue): D140-D144.

34. Cimmino A, Calin GA, Fabbri M, lorio MV, Ferracin M, Shimizu M et al. miR-15 and miR-16 induce apoptosis by targeting BCL2. Proc Natl Acad Sci USA 2005; 102: 3944-3949.

35. Volinia S, Calin GA, Liu CG, Ambs S, Cimmino A, Petrocca F et al. A microRNA expression signature of human solid tumors defines cancer gene targets. Proc Natl Acad Sci USA 2006; 103: 2257-2261.

36. Weiler J, Hunziker J, Hall J. Anti-miRNA oligonucleotides (AMOs): ammunition to target miRNAs implicated in human disease? Gene Therapy 2006; 13: 496-502.

37. Orom UA, Kauppinen S, Lund AH. LNA-modified oligonucleotides mediate specific inhibition of microRNA function. Gene 2006; 372: 137-141.

38. Krutzfeldt J, Kuwajima S, Braich R, Rajeev KG, Pena J, Tuschl T et al. Specificity, duplex degradation and subcellular localization of antagomirs. Nucleic Acids Res 2007; 35: 2885-2892.

39. Meng F, Henson R, Lang M, Wehbe H, Maheshwari S, Mendell JT et al. Involvement of human micro-RNA in growth and response to chemotherapy in human cholangiocarcinoma cell lines. Gastroenterology 2006; 130: 2113-2129.

Supplementary Information accompanies the paper on Cell Death and Differentiation website (http://www.nature.com/cdd) 